\title{
EARLY DETECTION OF RENAL BLOOD FLOW IMPEDANCE BY COLOUR DOPPLER IN CHRONIC LIVER DISEASE WITH NORMAL RENAL PARAMETERS
}

\author{
Dhurgesananthini Vijayasundaram¹, Kamatchi Karunanidhi², Banu Gopanar Balaraman³, Arjunan Senthil Kumaran 4
}

${ }^{1}$ Assistant Professor, Department of Medicine, Chengalpattu Medical College and Hospital.

${ }^{2}$ Assistant Professor, Department of Medicine, Chengalpattu Medical College and Hospital.

${ }^{3}$ Assistant Professor, Department of Medicine, Chengalpattu Medical College and Hospital.

${ }^{4}$ Assistant Professor, Department of Medicine, Chengalpattu Medical College and Hospital.

\section{ABSTRACT}

\section{BACKGROUND}

Chronic liver disease in the clinical context is a disease process of the liver that involves a process of progressive destruction and regeneration of the liver parenchyma leading to fibrosis and cirrhosis. Of the various complications in liver cirrhosis including hepatorenal syndrome type 1 , still carries the worst prognosis.

The aim of this study is to assess the resistivity index of cirrhotics with ascites and cirrhotics without ascites, to correlate with clinical status and serum creatinine levels, to detect persons with increased resistive index in the presence of normal renal parameters and to identify a subset of patients at higher risk of development of HRS to find out the usefulness and applicability of this methodology.

\section{MATERIALS AND METHODS}

A total of 92 patients in which there were 75 cases which included cirrhosis with ascites, cirrhosis without ascites and 17 controls which included fatty liver and healthy individuals formed the study group. Patients were selected for the study who satisfied all the inclusion and exclusion criteria. Study design is a single centre, descriptive, cross-sectional and non-interventional study. Inclusion criteria is patients attending Medical Gastroenterology Clinic and General Medicine Wards with Chronic Liver Disease with or without decompensation. Exclusion criteria is pre-existing renal parenchymal disease, other causes of renal vasoconstriction, druginduced renal failure (e.g. aminoglycosides and NSAIDs), glomerulonephritis, prerenal failure, sepsis and acute tubular necrosis. Detailed clinical history was taken from each patient and a complete review of their case notes performed. A complete clinical examination of the abdomen was done for each patient. Blood investigations were done. USG abdomen was done which detects the liver echoes, ascitic fluid and kidney size. Portal Doppler was done to elicit portal hypertension picture. Renal Doppler was done to measure the resistivity index. The RI of three renal vessels, obtained in three renal areas was measured in each patient by using at least three Doppler spectra and the mean value was calculated.

\section{RESULTS}

In our study in cirrhotics, renal vascular resistance indices evaluated by duplex Doppler ultrasonography are already increased in the early phase of the disease. Development of ascites is associated with a further increase in the resistance indices. Renal vasoconstriction evaluated by these indices is correlated with Child score, which quantitatively measures the hepatic function in cirrhosis.

\section{CONCLUSION}

We conclude that intrarenal RI measurement is a predictor of renal vasoconstriction and serves to detect early renal function impairment in cirrhotic patients. The diagnosis of elevated RI may be taken into account in the clinical management of these patients.

\section{KEYWORDS}

Chronic Liver Disease, Hepatorenal Syndrome, Resistivity Index.

HOW TO CITE THIS ARTICLE: Vijayasundaram D, Karunanidhi K, Balaraman BG, et al. Early detection of renal blood flow impedance by colour Doppler in chronic liver disease with normal renal parameters. J. Evolution Med. Dent. Sci. 2017;6(23):19241927, DOI: $10.14260 /$ Jemds/2017/422

\section{BACKGROUND}

Chronic liver disease in the clinical context is a disease process of the liver that involves a process of progressive destruction and regeneration of the liver parenchyma leading

Financial or Other, Competing Interest: None.

Submission 09-02-2017, Peer Review 07-03-2017,

Acceptance 13-03-2017, Published 20-03-2017.

Corresponding Author:

Dhurgesananthini Vijayasundaram,

Assistant Professor,

Department of Medicine,

Chengalpattu Medical College and Hospital.

E-mail: drdhurgesa76@yahoo.com

DOI: $10.14260 /$ jemds $/ 2017 / 422$

(c) (i) $\odot$ to fibrosis and cirrhosis. Cirrhosis is a consequence of chronic liver disease characterised by replacement of liver tissue by fibrosis, scar tissue and regenerative nodules (lumps that occur as a result of a process in which damaged tissue is regenerated) ${ }^{1,2}$ leading to loss of liver function. Cirrhosis is most commonly caused by alcoholism, hepatitis B and C and fatty liver disease, but has many other possible causes. Some cases are idiopathic, i.e. of unknown cause. Ascites (fluid retention in the abdominal cavity) is the most common complication of cirrhosis and is associated with a poor quality of life, increased risk of infection and a poor long-term outcome. Other potentially life-threatening complications are hepatic encephalopathy (confusion and coma) and bleeding from oesophageal varices. Cirrhosis is generally irreversible, 
and treatment usually focuses on preventing progression and complications. In advanced stages of cirrhosis, the only option is a liver transplant. The severity of cirrhosis is commonly classified with the Child-Pugh score. This score uses bilirubin, albumin, INR, presence and severity of ascites and encephalopathy to classify patients in class A, B or C; class $A$ has favourable prognosis, while class $C$ is at high risk of death. It was devised in 1964 by Child and Turcotte and modified in 1973 by Pugh et al. ${ }^{3}$

Of the various complications in liver cirrhosis including gastrointestinal bleeding, ascites and hepatocellular carcinoma, the rapidly progressive form of kidney dysfunction in cirrhosis, i.e. hepatorenal syndrome type 1, still carries the worst prognosis. In the early 1990s, median survival of these patients was reported to be as short as two weeks ${ }^{4}$ and some more recent papers indicate that in terms of prognosis there has not been much progress till now. ${ }^{5}$ However, during the past two decades, new treatment concepts based on an improved path physiological understanding of the mechanisms ultimately leading to Hepatorenal Syndrome (HRS) have been introduced. Despite successful drug treatment approaches to date, the only definitive treatment of HRS type 1 is liver transplantation or even combined liver/kidney transplantation in some patients. The annual incidence of hepatorenal syndrome among adults with ascites and cirrhosis is approximately $8 \%$. In addition, among adults with cirrhosis and portal hypertension, 20\% develop hepatorenal syndrome in the first year after diagnosis, and as many as $40 \%$ of patients develop hepatorenal syndrome within 5 years after diagnosis. ${ }^{6}$

Hepatorenal Syndrome (HRS) is characterised by renal vasoconstriction. ${ }^{7}$ Renal vasoconstriction has been documented in several series of cirrhotic patients by Doppler Ultrasound (US) analysis of renal arteries showing increased Resistive Index (RI), ${ }^{8}$ which is determined from the spectral waveforms and corresponds to the following formula: (peak systolic frequency shift- lowest diastolic frequency shift)/peak systolic frequency shift.

On average, renal RI is higher in cirrhotic patients compared to healthy individuals and high RI (over 0.7 ) can be observed in cirrhotic patients with serum creatinine within the normal range. In patients without refractory ascites RI decreases from the hilum towards the outer parenchyma, suggesting that the flow to the cortex is relatively preserved. In contrast in patients with refractory ascites, RI is also increased in the cortical vessels suggesting cortical vasoconstriction. Paracentesis and albumin infusion are followed by a significant decrease in renal RI. ${ }^{9}$ Liver transplantation is also followed by a decrease in RI.10

The aim of this study is to assess the resistivity index of cirrhotics with ascites and cirrhotics without ascites, to correlate with clinical status and serum creatinine levels, to detect persons with increased resistive index in the presence of normal renal parameters and to identify a subset of patients at higher risk of development of HRS, to find out the usefulness and applicability of this methodology.

\section{MATERIALS AND METHODS}

A total of 92 patients in which there were 75 cases which included cirrhosis with ascites, cirrhosis without ascites and 17 controls which included fatty liver and healthy individuals formed the study group. Patients were selected for the study who satisfied all the inclusion and exclusion criteria. Written consent was obtained from all the patients participating in the study. All patients and controls were not from a single ethnic background. Study design is a Single centre, descriptive, cross-sectional, non-interventional study. Comparisons were performed by t-test. Correlation was done by Pearson's Correlation Coefficient.

Inclusion criteria is patients attending Medical Gastroenterology Clinic and General Medicine Wards with Chronic Liver Disease with or without decompensation.

Exclusion criteria is preexisting renal parenchymal disease, other causes of renal vasoconstriction, drug-induced renal failure (e.g. amino-glycosides and NSAIDs), glomerulonephritis, pre-renal failure, sepsis and acute tubular necrosis.

Detailed clinical history was taken from each patient and a complete review of their case notes performed. A complete clinical examination of the abdomen was done for each patient. Blood investigations were done. USG abdomen was done which detects the liver echoes, ascitic fluid and kidney size. Portal Doppler was done to elicit portal hypertension picture. Renal Doppler was done to measure the resistivity index. The RI of three renal vessels obtained in three renal areas was measured in each patient by using at least three Doppler spectra and the mean value was calculated.

\section{RESULTS}

\section{Age Wise Classification}

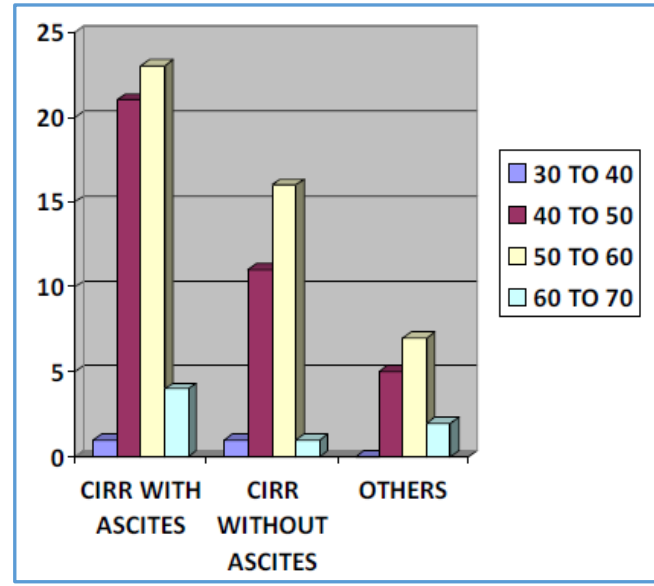

Figure 1. Among 92, 25\% of Cirrhosis with Ascites Patients Falls in the Age Group of 50 to 60 Years, 23\% are in the Age Group of 40 to 50 Years

\section{Sex Wise Classification}

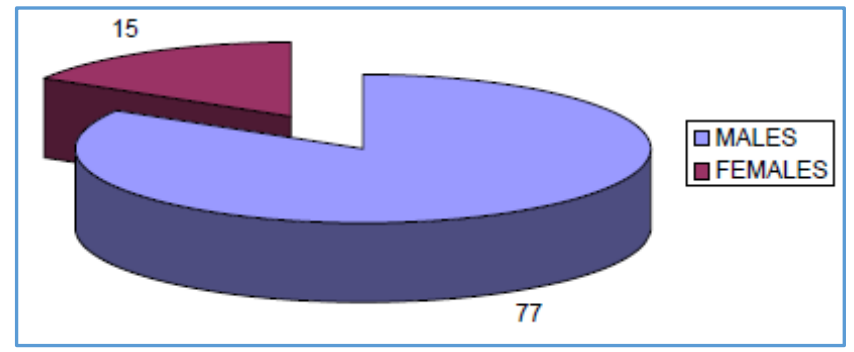

Figure 2. Out of the Total of 92 patients 77 were Males, 15 were Females. In the above mentioned Study, $67 \%$ were Males compared to our Study which shows $84 \%$ Males. Alcoholic vs Non-Alcoholic 


\begin{tabular}{|c|c|c|}
\hline & Males & Females \\
\hline Alcoholic & $48(52 \%)$ & $07(8 \%)$ \\
\hline Non-Alcoholic & $19(21 \%)$ & $06(6 \%)$ \\
\hline Healthy & $10(11 \%)$ & $02(2 \%)$ \\
\hline $\begin{array}{r}\text { Table 1. Out of the 77 Males 52\% were Alcoholic and 8\% of } \\
\text { Females also were Alcoholic }\end{array}$
\end{tabular}

\begin{tabular}{|c|c|}
\hline Cirrhosis/Ascites & $49(53 \%)$ \\
\hline Cirrhosis/Non-Ascites & $26(28 \%)$ \\
\hline Healthy & $17(18 \%)$ \\
\hline
\end{tabular}

Table 2. Total 92 Patients, 49 were Cirrhosis with Ascites patients, 26 were Cirrhosis without Ascites patients, 17 Healthy which includes Fatty Liver Also

\section{Classification Based on Causes}

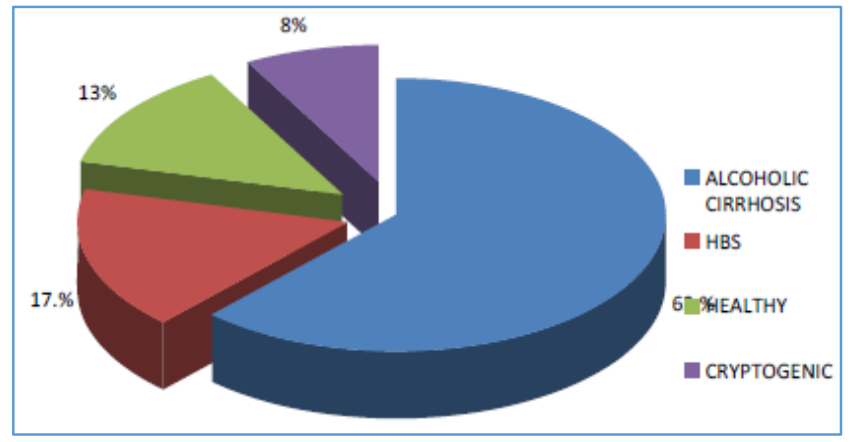

Figure 3. Alcoholic Cirrhosis is $62 \%, 17 \%$ were Hepatitis B Positive and 8\% were of Cryptogenic Cause

\section{Resistivity Index in Various Groups}

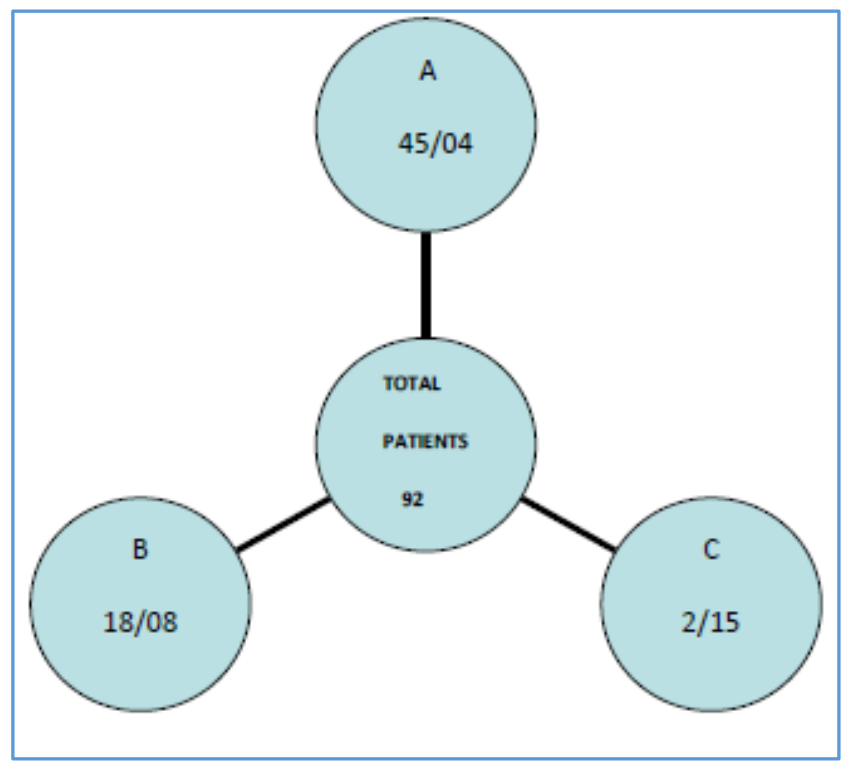

Figure 4. Showing RI in various Groups

\begin{tabular}{|c|c|c|c|}
\hline Groups & Categories & $\begin{array}{c}\text { Elevates } \\
\text { RI }\end{array}$ & $\begin{array}{c}\text { Normal } \\
\text { RI }\end{array}$ \\
\hline A & Cirrhosis/Ascites & 45 & 04 \\
\hline B & $\begin{array}{c}\text { Cirrhosis/Non- } \\
\text { Ascites }\end{array}$ & 18 & 08 \\
\hline C & Fatty Liver/Healthy & 02 & 15 \\
\hline \multicolumn{2}{|c|}{ Table 3. Showing RI in various Groups. Elevated RI } \\
65/92=71\%. Normal RI 27/92=29\% \\
\hline
\end{tabular}

Comparing Resistivity Indices

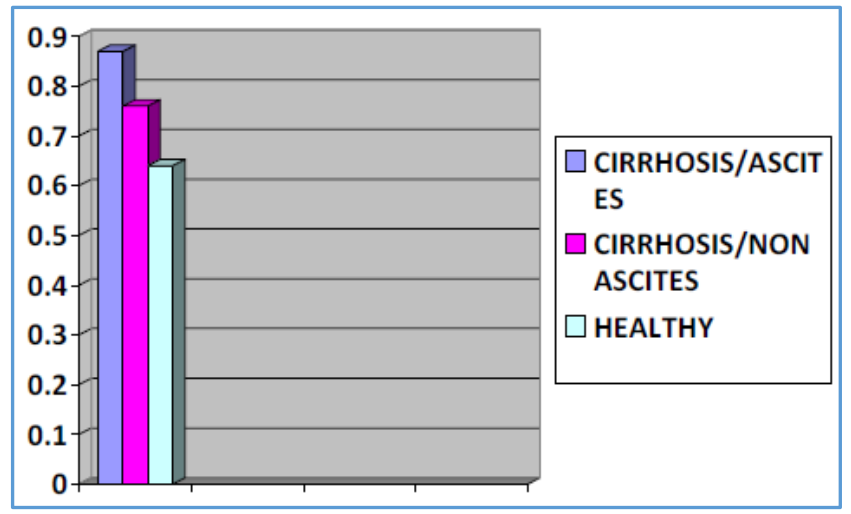

Figure 5. A Renal RI of 0.70 or more was considered Abnormal according to the Study of Platt JF, Ellis JH, Rubin JM, Merion RM, Lucey MR: Renal Duplex Doppler Ultrasonography: A Non-Invasive Predictor of Kidney Dysfunction Hepatorenal Failure in Liver Disease. Hepatology 20:362-369, 199411

In our study (Figure 13), Resistivity index is increased in both cirrhosis with ascites and without ascites. So early phase itself shows increased RI. In Eur J Med Res. 2008 Aug 18;13(8):383-7, RI was significantly higher in ascitic patients compared to non-ascitic patients ( 0.74 vs. $0.67, \mathrm{p}<0.01)$ and in non-ascitic patients with liver cirrhosis than in control subjects $(0.67$ vs. $0.62, \mathrm{p}<0.01)$.

\section{Probability of HRS in Cirrhosis with Ascites}

\begin{tabular}{|c|c|}
\hline Cirrhosis with Ascites & 49 Patients \\
\hline Probability of Hepatorenal & 13 Patients \\
\hline Table 4. Considering various Studies, the Probability \\
of HRS in Cirrhosis with Ascites is 26\% \\
\hline
\end{tabular}

In our study, RI was significantly higher in ascitic patients compared to non-ascitic patients $(0.87$ vs. $0.76, \mathrm{p}<0.01)$ and in non-ascitic patients with liver cirrhosis than in control subjects ( 0.76 vs. $0.64, \mathrm{p}<0.001$ ).

\section{DISCUSSION}

As majority of individuals with hepatorenal syndrome have cirrhosis, much of the epidemiological data on HRS comes from the cirrhotic population. The condition is quite common: approximately $10 \%$ of individuals admitted to hospital with ascites have HRS. A retrospective case series of cirrhotic patients treated with terlipressin suggested that $20.0 \%$ of acute renal failure in cirrhotics was due to type $1 \mathrm{HRS}$ and $6.6 \%$ was due to type 2 HRS. It is estimated that $18 \%$ of individuals with cirrhosis and ascites will develop HRS within one year of their diagnosis with cirrhosis and 39\% of these individuals will develop HRS within five years of diagnosis. The mean age was $51.8+/-12.1$ years and $67 \%$ were male as per the study. In our study 92 patients were studied including cirrhosis with ascites (49) without ascites (26), fatty liver and healthy individuals (17).

Many of them are in the fourth (40\%) and fifth (51\%) decades. Males (84\%) are more common than females $(16 \%)$ $60 \%$ were alcoholic and $25 \%$ were non-alcoholic. Of the various causes apart from alcoholic, $17 \%$ were hepatitis $\mathrm{B}$ positive, $8 \%$ were cryptogenic and $13 \%$ were healthy. 
In our study 49 (53\%) were cirrhosis with ascites patients, $26(28 \%)$ were cirrhosis without ascites patients, 17 (18\%) healthy which includes fatty liver also. Doppler sonography is performed to determine the intrarenal arteriolar vascular resistance defined as the resistive index (RI). The reference range for RI is 0.7 or lower. The RI can be a more sensitive parameter than the creatinine clearance. Renal duplex Doppler ultrasonography can non-invasively identify a subgroup of non-azotemic patients with liver disease that is at significantly higher risk for subsequent development of kidney dysfunction and the hepatorenal syndrome.

Out of the 49 cirrhosis with ascites patients, 45 were showing elevated resistivity index, 4 were within normal range. Among 26 cirrhosis without ascites patients, 18 were showing elevated resistivity index, 8 were within normal range. Resistivity indices were increased in both cirrhosis with and without ascites. Cirrhosis with ascites $=>0.87$ Cirrhosis without ascites $=>0.76$ Fatty liver and healthy $=>$ 0.64. In the study by Eur J Med Res. 2008 Aug 18;13(8):383-7, RI was significantly higher in ascitic patients compared to non-ascitic patients (0.74 vs. $0.67, \mathrm{p}<0.01)$ and in non-ascitic patients with liver cirrhosis than in control subjects $(0.67$ vs. $0.62, \mathrm{p}<0.01$ ).

In our study, RI was significantly higher in ascitic patients compared to non-ascitic patients $(0.87$ vs. $0.76, p<0.01)$ and in non-ascitic patients with liver cirrhosis than in control subjects $(0.76$ vs. $0.64, \mathrm{p}<0.001)$. Renal vascular resistance indices evaluated by duplex Doppler ultrasonography are already increased in the early phase of the disease.

Development of ascites is associated with a further increase in the resistance indices. Renal vasoconstriction evaluated by these indices is correlated with Child score, which quantitatively measures the hepatic function in cirrhosis. PI is also increased in cirrhosis with ascites patients compared to cirrhotics without ascites (1.12 vs. 1.02), cirrhotics without ascites compared to healthy (1.02 vs. 0.08); $26 \%$ of cirrhosis with ascites patients later tends to develop HRS; 55\% cirrhosis with elevated RI and normal renal function develops subsequent kidney dysfunction that is 35 patients out of 92 patients.

We conclude that patients with cirrhosis are at risk of renal deterioration which cannot be detected by serum urea, creatinine and glomerular filtration rate. The increase of RI is associated with the progress of hepatocellular disease and also the development of ascites and portal hypertension. Hence, monitoring RI is a non-invasive means of studying early renal haemodynamic alteration in cirrhosis.

\section{CONCLUSION}

1. We conclude that in cirrhotic, renal vascular resistance indices evaluated by duplex Doppler ultrasonography are already increased in the early phase of the disease.
2. Development of ascites is associated with a further increase in the resistance indices.

3. Renal vasoconstriction evaluated by these indices is correlated with Child score, which quantitatively measures the hepatic function in cirrhosis.

4. Intrarenal RI measurement is a predictor of renal vasoconstriction and serves to detect early renal function impairment in cirrhotic patients.

5. The diagnosis of elevated RI may be taken into account in the clinical management of these patients.

6. The limitation of the study is small sample size.

\section{REFERENCES}

[1] Liver cirrhosis. Review of pathology of the liver. http://www.meddean.luc.edu/lumen/MedEd/orfpath /cirhosis.htm.

[2] Pathology education: gastrointestinal. http://www.pathology.vcu.edu/education/gi/lab3.h.h tml.

[3] Pugh RN, Murray-Lyon IM, Dawson JL, et al. Transection of the oesophagus for bleeding oesophageal varices. Br J Surg 1973;60(8):646-9.

[4] Gines P, Guevara M, Arroyo V, et al. Hepatorenal syndrome. Lancet 2003;362(9398):1819-27.

[5] Alessandria C, Debernardi-Venon W, Carello M, et al. Midodrine in the prevention of hepatorenal syndrome type 2 recurrence: a case-control study. Dig Liver Dis Apr 2009;41(4):298-302.

[6] Xu X, Ling Q, Zhang M, et al. Outcome of patients with hepatorenal syndrome type 1 after liver transplantation: Hangzhou experience. Transplantation 2009;87(10):1514-9.

[7] Fernandez-Seara J, Prieto J, Quiroga J, et al. Systemic and regional hemodynamics in patients with liver cirrhosis and ascites with and without functional renal failure. Gastroenterology 1989;97(5):1304-12.

[8] Kastelan S, Ljubicic N, Kastelan Z, et al. The role of duplex-doppler ultrasonography in the diagnosis of renal dysfunction and hepatorenal syndrome in patients with liver cirrhosis. Hepatogastroenterology 2004;51(59):1408-12.

[9] Bardi A, Sapunar J, Oksenberg D, et al. Intrarenal arterial doppler ultrasonography in cirrhotic patients with ascites, with and without hepatorenal syndrome. Rev Med Chil 2002;130(2):173-80.

[10] Unceta RP, Mangas RA, Rodríguez MMA, et al. Doppler ultrasonography in the assessment of renal hemodynamics in patients with chronic liver disease. Rev Esp Enferm Dig 2000;92(12):799-805.

[11] Platt JF, Ellis JH, Rubin JM, et al. Renal duplex doppler ultrasonography: a noninvasive predictor of kidney dysfunction hepatorenal failure in liver disease. Hepatology 1994;20(2):362-9. 